\title{
Compact, astigmatism corrected cross-grating spectrometer
}

\author{
Matthias Kraus ${ }^{1,2, *}$, Erik Förster ${ }^{1,2}$, Verena Bagusat $^{1}$, Tobias Hönle ${ }^{1}$, Xavier Uwurukundo ${ }^{1}$, Patrick Bohnert $^{1}$, Robert \\ Brüning $^{3}$, Hartmut Hillmer ${ }^{2}$, and Robert Brunner ${ }^{1,3}$ \\ ${ }^{1}$ University of Applied Sciences Jena, 07745 Jena, Germany \\ ${ }^{2}$ Institute of Nanostructure Technologies and Analytics (INA), University of Kassel, 34132 Kassel, Germany \\ ${ }^{3}$ Fraunhofer Institute for Applied Optics and Precision Engineering (IOF), 07745 Jena, Germany
}

\begin{abstract}
Compact cross-grating spectrometers are inspired by classical high-end Echelle spectrometers and allow to acquire a large spectrum with a high spectral resolution in a single shot. To decrease the size of an Echelle spectrometer substantially, a cross grating was employed combining both required dispersive functionalities. First, an Echelle grating diffracts light in several higher diffraction orders, while a superposed, perpendicularly oriented cross-disperser is used in first diffraction order. In this contribution, we report on the realization of a cross-grating spectrometer employing a folded reflective beam path. A toric-convex mirror was introduced allowing aberration compensation. We present the basic concept and optical design of the system and discuss the mechanical implementation and the adjustment process. Here, different laser sources and gas emission lamps are used to realize the system and derive the optical performance. In particular, the spectral resolution in the different diffraction orders is determined for the realized device.
\end{abstract}

\section{Introduction}

The market for spectroscopy is known as a growing market based on an increasing number of applications, for example in industrial process control, agriculture or environmental monitoring. These new applications are connected to increasing demands on the spectroscopic instruments itself. In particular, compact devices without moving parts are demanded which allow to acquire simultaneously a wide spectral range with high resolution. It is known that these specifications are contradictory and cannot be fulfilled by established compact spectrometers based on a concave grating mount. Classical Echelle spectrometers are able to measure a large spectral bandwidth with an extremely high resolution. However, they require a large volume and are very expensive [1]. Echelle-inspired cross-grating spectrometers are able to bridge the gap between these two instrumental concepts [2-4]. The cross-grating as the key element combines two dispersive functionalities in a single element which are separated in classical Echelle spectrometers. Therefore, it consists of two superposed blazed line gratings: The Echelle grating and the cross-dispersion grating. The Echelle grating is used in moderately higher diffraction orders (here $4^{\text {th }}$ to $11^{\text {th }}$ ) and diffracts the incoming light into different overlapping spectral bands with a high spectral resolution. These overlapping diffraction orders are separated by the perpendicularly oriented crossdispersion grating which is used in $1^{\text {st }}$ diffraction order. The use of the combined cross-grating allows a substantial size reduction compared to classical Echelle spectrometers but requires new optical design approaches.
In this contribution, we report on the realization of a compact cross-grating spectrometer which covers a spectral range from $330 \mathrm{~nm}$ to $1100 \mathrm{~nm}$ with a resolving power $\lambda / \Delta \lambda$ higher than 500 . As a starting point, we present the working principle and the optical design comprising a folded, all-reflective beam-path including a toric-convex mirror used for aberration compensation. Afterwards, we present the mechanical layout and discuss aspects of the system mounting and the adjustment process. Furthermore, optical key parameters like the spectral resolution are determined by measurements.

\section{Optical design and implementation}

The optical design is based on a folded Czerny-Turner setup with an additional toric correction mirror [3]. The light is fed to the spectrometer by an optical fiber which works as the entrance pinhole. The divergent beam is collimated by a concave mirror and propagates to a toric correction mirror for aberration compensation. In particular, astigmatism has to be corrected which is introduced to the system by the subsequent following cross-grating. It is illuminated at an oblique angle of 22.9 degree and diffracts the light in two orthogonal directions. The light propagates to the focusing mirror and is imaged onto the 2D detector. Corresponding to the separated diffraction orders, linear spectral bands are formed on the detector. Due to the spectral sensitivity of the siliconbased detector, the detectable spectrum ranges from approximately $330 \mathrm{~nm}$ to $1100 \mathrm{~nm}$. For the mechanical design of the prototype, standard catalogue elements are

\footnotetext{
* Corresponding author: Matthias.Kraus@eah-jena.de
} 
preferentially used. This allows an inexpensive setup at the cost of slightly increasing space demands. Figure 1 shows a photograph of the realized setup. The full system is attached to a stable base plate which is part of the stray light reducing black housing.

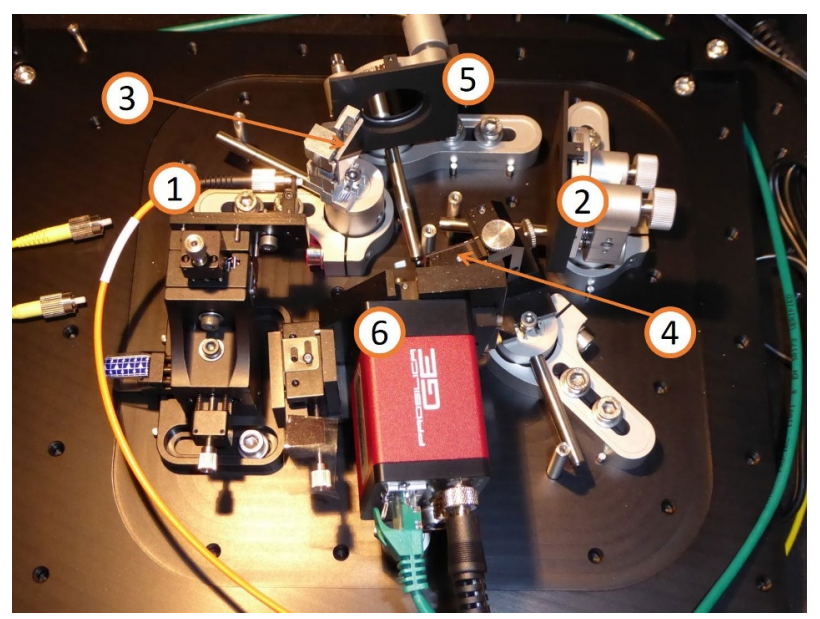

Fig. 1. Photograph of the realized spectrometer. The different mounted components are labelled with the following numbers: fiber (1), collimating mirror (2), toric correction mirror (3), cross-grating (4), focusing mirror (5) and detector (6).

The light is lead into the housing through the optical fiber and is collimated by a spherical mirror and a toric correction mirror. The mirror's radii of curvature are infinity in one direction and approximately $12 \mathrm{~m}$ in the other direction. In order to provide a mirror with the required sag height of about $4 \mu \mathrm{m}$, we deformed a plane mirror in a precise bending mount. The resulting sag height is controlled with a Fizeau-Interferometer.

In the beam-path follows the cross-grating which is a superposition of two blazed gratings and is manufactured by direct laser beam writing. The Echelle grating has a period of $10 \mu \mathrm{m}$ and a height of $1.5 \mu \mathrm{m}$ while the perpendicularly oriented cross dispersion grating has a period of $5.2 \mu \mathrm{m}$ and a height of $150 \mathrm{~nm}$ [4]. The light, which is diffracted by the cross-grating, is imaged onto a monochrome camera (Allied Vision, GE2040) by the focusing mirror. In order to determine the spectral resolution and precise wavelength range for each diffraction order, different gas emission lamps $(\mathrm{HgCd}$, $\mathrm{HgAr}, \mathrm{Na}, \mathrm{Cs}$ ) were used. We measured the pixel distance between known spectral lines and the full width half maximum (FWHM) size of each spectral line. Based on the ratio of pixel distance to wavelength distance between these lines, the covered spectral range and spectral resolution for all diffraction orders can be derived. We determined a resolving power $\lambda / \Delta \lambda$ higher than 500 for the full spectral range. The central wavelength range, which is imaged with low aberrations on the detector, an even higher resolving power of more than 800 is obtained. Figure 2 shows an acquired spectrum of a HgAr-lamp. The measured intensity distribution shows distinct spectral lines in different diffraction orders $\left(4^{\text {th }}\right.$ to $6^{\text {th }}$ diffraction order of the Echelle grating) which are labelled with table values [5]. The inset shows a magnified view of specific spectral lines. The wavelength pair
$810.37 / 811.53 \mathrm{~nm}$ are clearly separated. This corresponds to a resolving power of $\sim 700$.

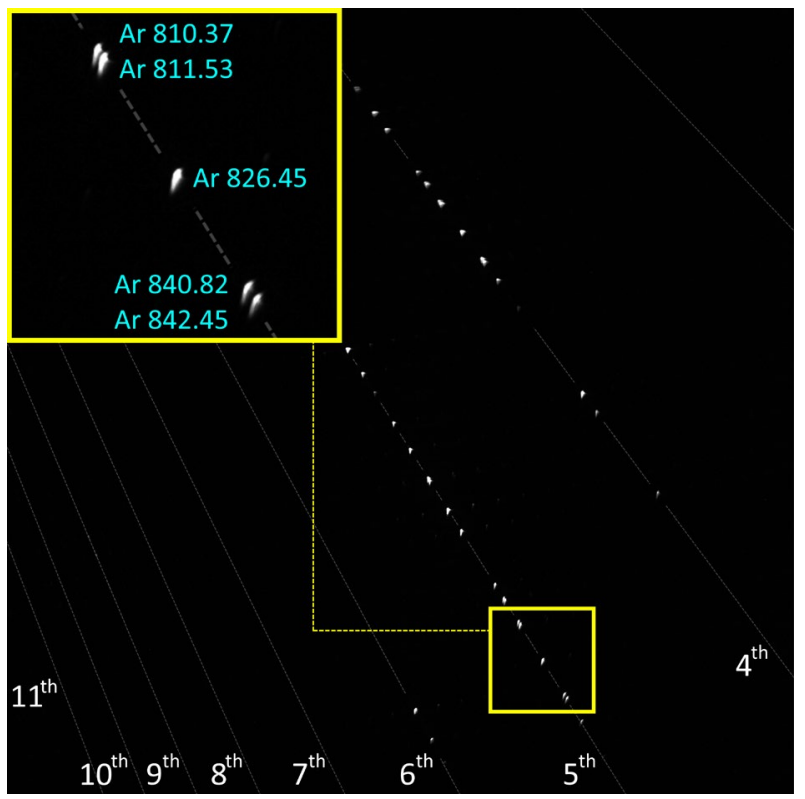

Fig. 2. Acquired line spectra of a HgAr-lamp. Spectral lines appear in the $4^{\text {th }}$ to the $6^{\text {th }}$ diffraction order. The image positions of all usable diffraction orders are marked by dashed lines. The inset shows a magnified view of specific spectral lines.

\section{Conclusion}

This contribution presents the realization of a compact cross-grating spectrometer with an all-reflective, folded beam path. The utilized cross-grating is a superposition of two blazed line gratings and allows to address a wide spectral range with a high resolution. Starting from the basic concept and the optical design, we report on the mechanical layout and the experimental demonstration of the setup. We proved by measurements that the realized setup covers the spectral range from $330 \mathrm{~nm}$ to $1100 \mathrm{~nm}$ and allows a resolving power of more than 500 .

\section{References}

1. T. Eversberg, K. Vollmann, Spectroscopic Instrumentation, (Springer, Heidelberg, 2015)

2. D. Thomae, T. Hönle, M. Kraus, V. Bagusat, A. Deparnay, R. Brüning, R. Brunner, Appl. Opt. 57(25), 7109-7116 (2018)

3. V. Bagusat, M. Kraus, E. Förster, D. Thomae, T. Hönle, R. Brüning, H. Hillmer, R. Brunner, J. Opt. Soc. Am. A 36(3), 345-352 (2019)

4. M. Kraus, E. Förster, V. Bagusat, T. Hönle, X. Uwurukundo, P. Bohnert, R. Brüning, H. Hillmer, R. Brunner, Appl. Opt. 59(5), 1338-1346 (2020)

5. Kramida, A., Ralchenko, Yu., Reader, J., and NIST ASD Team (2019). NIST Atomic Spectra Database (ver. 5.7.1), [Online]. Available: https://physics.nist.gov/asd [2020, March 2]. 